\title{
Un modelo SIR para el estudio de la Rickettsiosis
}

\author{
Gabriel Adrián Salcedo Varela \\ David Baca Carrasco \\ Universidad de Sonora \\ e-mail: adrian_salcedo100393@hotmail.com \\ davidbaca@mat.uson.mx
}

\begin{abstract}
Resumen
En el presente trabajo, se presenta un modelo matemático mediante el cual se está estudiando la zoonosis conocida como Rickettsiosis. Dicha enfermedad está presente en distintos parásitos como los piojos, pulgas y las garrapatas. La Rickettsiosis se ha presentado en muchos lugares del mundo a lo largo de la historia, en particular, en los últimos años se ha estado presentando con mucha frecuencia en el estado de Sonora. Nuestro interés, es estudiar la dinámica de la propagación de la Rickettsiosis, así como la de buscar medidas sanitarias que permitan reducir o controlar su propagación. Para ello, se calcula y se analiza el número reproductivo básico, denotado por $R_{0}$, para el modelo propuesto, de tal manera que su análisis nos permite entender cuáles son los factores que propician la propagación y con ello, nos permite proponer algunas medidas de control.
\end{abstract}

\section{Introducción}

La Rickettsiosis es una zoonosis de incidencia mundial. Es causada por bacterias del género Rickettsia, de las cuales existen muchas cepas, de entre las cuales podemos destacar, por su infecciosidad, la rickettsia rickettsii, rickettsia prowazekii y la rickettsia typhi, siendo para el humano la rickettsia rickettsii la más agresiva y motivo de estudio en este trabajo. Las rickettsias son bacterias intracelulares obligadas, no forman esporas y son Gram-negativas.

Los principales reservorios de las rickettsias son el piojo, la pulga y la garrapata. En el caso de rickettsia rickettsii, su principal reservorio es la garrapata (Dermacentor variables), y que a su vez, su principal hospedero es el perro doméstico, lo cual propicia el contacto con el humano [1].

La transmisión de la enfermedad, tanto al humano (hospedero accidental) como al perro (hospedero secundario), se da a través de la picadura de la garrapata infectada, la cual al alimentarse, transfiere la bacteria a través de la saliva. De manera análoga, una garrapata sana se puede infectar a través de un perro infectado de rickettsiosis al alimentarse de él. Por otro lado, el ciclo de vida de las garrapatas se da a través de 4 etapas: huevos, larva, ninfa y adulta y en cualquiera de las etapas a partir de larva, la garrapata puede infectar y ser infectada. En general, un ataque confiere inmunidad permanente, es decir, una vez que te recuperas de la enfermedad, ya no vuelves a contarerla de nuevo [2].

En el humano, los síntomas principales de la rickettsiosis son: mialgias, fiebre, escalofríos, cefalea, dolor abdominal, náuseas, vómito, diarrea, anorexia, tos, entre otros [3]. El periodo 
de incubación de la bacteria es de 14 días y si no se trata a tiempo, la enfermedad puede causar la muerte [3].

Desde los años 40 se han reportado casos de rickettsiosis en varios estados de la república. De manera particular, durante el año 2014, en Sonora se reportaron 89 casos de rickettsiosis, de los cuales 11 terminaron en deceso. Por esta razón, estamos interesados en estudiar la propagación de la enfermedad, pensando en proponer medidas de control para disminuir la incidencia de dicha enfermedad en el estado de Sonora.

Es por ello que este trabajo está desglosado de la siguiente manera. En la sección 2, presentamos un modelo matemático para estudiar la dinámica de propagación de la enfermedad de la rickettsiosis. Dicho modelo representa las características básicas de las poblaciones que participan en la dinámica de propagación (humanos, perros y garrapatas). En la sección 3 se hace un análisis de estabilidad de los puntos de equilibrio del sistema, en la sección 4 se calcula y analiza el número reproductivo básico y con ello se proponen medidas de control para la enfermedad.

En la Figura 1 se muestra el ciclo de vida de la rickettsia rickettsii.

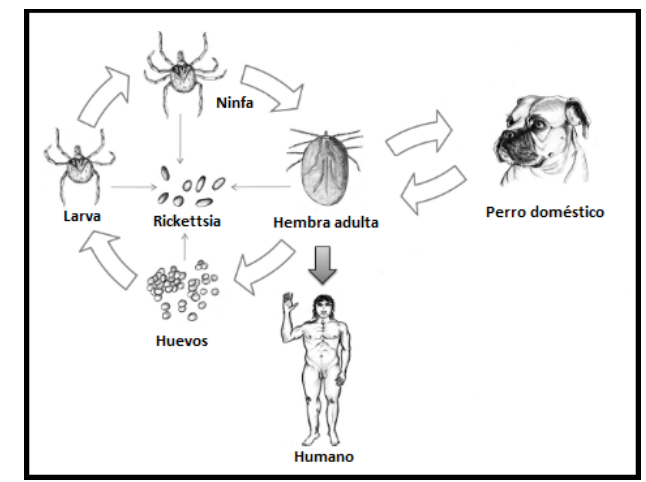

Figura 1: Ciclo de vida de la rickettsia. Figura tomada de [2].

La figura 1, nos muestra la dinámica completa de cómo se da la propagacin de la enfermedad en el humano y en el perro, considerando las distintas etapas de vida de la garrapata. Sin embargo, para los fines de este trabajo, en la sección 2, consideramos una dinámica de transmisin más simple, pero que describe de manera clara el ciclo de infección de la enfermedad.

\section{Modelo SIR para la Rickettsiosis}

En está sección, construiremos un modelo matemático que describe el ciclo de infección de la rickettsiosis entre las especies que participan, con el cual vamos a estudiar la dinámica de propagación de dicha enfermedad. Por simplicidad, para el desarrollo de nuestro modelo, no distinguiremos las distintas etapas de crecimiento de la garrapata; las englobaremos en una sola clase. Por lo tanto, el ciclo de infección de la rickettsia rickettsii considerado para el modelo es el siguiente: 


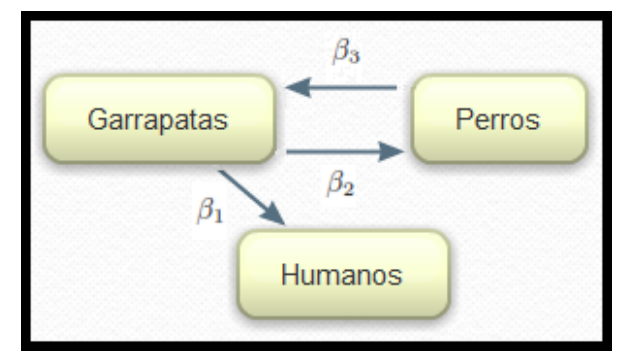

Figura 2: Ciclo de infección de la rickettsia rickettsii.

De este diagrama, las ecuaciones para el modelo son:

$$
\begin{aligned}
\dot{S_{H}} & =-\beta_{1} S_{H} I_{G}-\mu_{1} S_{H}+k_{1} \\
\dot{I_{H}} & =\beta_{1} S_{H} I_{G}-\left(\mu_{1}+\alpha_{1}+\gamma_{1}\right) I_{H} \\
\dot{R_{H}} & =\gamma_{1} I_{H}-\mu_{1} R_{H} \\
\dot{S_{P}} & =-\beta_{2} S_{P} I_{G}-\mu_{2} S_{P}+k_{2} \\
\dot{I_{P}} & =\beta_{2} S_{P} I_{G}-\left(\mu_{2}+\alpha_{2}\right) I_{P} \\
\dot{S_{G}} & =-\beta_{3} S_{G} I_{P}-\mu_{3} S_{G}+k_{3} \\
\dot{I_{G}} & =\beta_{3} S_{G} I_{P}-\mu_{3} I_{G} .
\end{aligned}
$$

Donde $S_{H}, I_{H}$ y $R_{H}$ representan las poblaciones de humanos susceptibles, infecciosos y recuperados respectivamente; $S_{P}$ e $I_{P}$ representan las poblaciones de perros susceptibles e infecciosos respectivamente; $S_{G}$ e $I_{G}$ son las poblaciones de garrapatas susceptibles e infecciosas; $\beta_{1}$ es la tasa de contagio de un humano susceptible al estar en contacto con una garrapata infectada, $\beta_{2}$ es la tasa de contagio de un perro susceptible al estar en contacto con una garrapata infectada, $\beta_{3}$ es la tasa de contagio de una garrapata susceptible al estar en contacto con un perro infectado, $k_{1}, k_{2}$ y $k_{3}$ son las tasas de entrada de los humanos, perros y garrapatas respectivamente, los cuales entran susceptibles a su respectiva clase; $\mu_{1}$, $\mu_{2}$ y $\mu_{3}$ representan las tasas de muerte natural de los humanos, perros y garrapatas respectivamente; $\alpha_{1}$ y $\alpha_{2}$ son las tasas de muerte por la enfermedad en los humanos y perros; $\gamma_{1}$ es la tasa de recuperación a la rickettsiosis de los humanos; $N_{H}, N_{P}$ y $N_{G}$ son las poblaciones totales de humanos, perros, garrapatas respectivamente, y son tales que $N_{H}=S_{H}+I_{H}+R_{H}$ y $N_{P}=S_{P}+I_{P}$ y $N_{G}=S_{G}+I_{G}$.

\section{Cálculo y análisis de estabilidad de los puntos de equilibrio del sistema}

En esta sección vamos a analizar la estabilidad de los puntos equilibrios del sistema (1), la cual está determinada por el signo de la parte real de los valores propios de la matriz jacobiana del sistema evaluada en dichos puntos de equilibrio.

Haciendo los cálculos correspondientes, se tiene que los puntos de equilibrio del sistema (1) están dados por: $x_{0}=\left(\frac{k_{1}}{\mu_{1}}, 0,0, \frac{k_{2}}{\mu_{2}}, 0, \frac{k_{3}}{\mu_{3}}, 0\right)$ y $x_{1}=\left(\frac{a_{1}}{a_{2}-a_{3}}, \frac{k_{1} a_{4}}{\left(a_{2}+a_{3}\right) a_{5}}, \frac{\beta_{1} \gamma_{1} k_{1} a_{6}}{a_{2} a_{5} \mu_{1}}, \frac{\mu_{3} a_{8}}{\beta_{3} a_{7}}, \frac{a_{6}}{\beta_{3} a_{7} a_{9}}, \frac{a_{7} a_{9}}{\beta_{2} a_{8}}, \frac{a_{6}}{\beta_{2} \mu_{3} a_{8}}\right)$, 
donde

- $a_{1}=\beta_{2} \beta_{3} k_{1} k_{2} \mu_{3}+\alpha_{2} \beta_{2} k_{1} \mu_{3}^{2}+\beta_{2} k_{1} \mu_{2} \mu_{3}^{2}$.

- $a_{2}=\beta_{1} \beta_{2} \beta_{3} k_{2} k_{3}+\beta_{2} \beta_{3} k_{2} \mu_{1} \mu_{3}+\alpha_{2} \beta_{2} \mu_{1} \mu_{3}^{2}-\alpha_{2} \beta_{1} \mu_{2} \mu_{3}^{2}+\beta_{2} \mu_{1} \mu_{2} \mu_{3}^{2}$.

- $a_{3}=\beta_{1} \mu_{2}^{2} \mu_{3}^{2}$.

- $a_{4}=\beta_{1} \beta_{2} \beta_{3} k_{2} k_{3}-\alpha_{2} \beta_{1} \mu_{2} \mu_{3}^{2}-\beta_{1} \mu_{2}^{2} \mu_{3}^{2}$.

- $a_{5}=\alpha_{1}+\mu_{1}+\gamma_{1}$.

- $a_{6}=\beta_{2} \beta_{3} k_{2} k_{3}-\alpha_{2} \mu_{2} \mu_{3}^{2}-\mu_{2}^{2} \mu_{3}^{2}$.

- $a_{7}=\beta_{2} k_{3}+\mu_{2} \mu_{3}$.

- $a_{8}=\beta_{3} k_{2}+\alpha_{2} \mu_{3}+\mu_{2} \mu_{3}$.

- $a_{9}=\alpha_{2}+\mu_{2}$.

3.1 Análisis de estabilidad del punto de equilibrio libre de infección

Para obtener la estabilidad del punto de equilibrio libre de infección, necesitaremos los valores propios de la matriz jacobiana evaluada en dicho punto. Así, para el sistema (1) la matriz jacobiana evaluada en el equilibrio libre de infección es la siguiente.

$$
J\left(x_{0}\right)=\left[\begin{array}{ccccccc}
-\mu_{1} & 0 & 0 & 0 & 0 & 0 & -\beta_{1} \frac{k_{1}}{\mu_{1}} \\
0 & -\left(\alpha_{1}+\mu_{1}+\gamma_{1}\right) & 0 & 0 & 0 & 0 & \beta_{1} \frac{k_{1}}{\mu_{1}} \\
0 & \gamma_{1} & -\mu_{1} & 0 & 0 & 0 & 0 \\
0 & 0 & 0 & -\mu_{2} & 0 & 0 & -\beta_{2} \frac{k_{2}}{\mu_{2}} \\
0 & 0 & 0 & 0 & -\left(\alpha_{2}+\mu_{2}\right) & 0 & \beta_{2} \frac{k_{2}}{\mu_{2}} \\
0 & 0 & 0 & 0 & -\beta_{3} \frac{k_{3}}{\mu_{3}} & -\mu_{3} & 0 \\
0 & 0 & 0 & 0 & \beta_{3} \frac{k_{3}}{\mu_{3}} & 0 & -\mu_{3}
\end{array}\right] .
$$

De esta manera, tenemos que los valores propios de la matriz (2) son:

- $\lambda_{1}=-\mu_{1}$

- $\lambda_{2}=-\mu_{2}$

- $\lambda_{3}=-\mu_{3}$

- $\lambda_{4}=-\left(\alpha_{1}+\mu_{1}+\gamma_{1}\right)$

- $\lambda_{5}=-\frac{\left(\alpha_{2}+\mu_{2}+\mu_{3}\right)}{2}-\frac{\sqrt{4 \beta_{2} \beta_{3} k_{2} k_{3}+\mu_{3} \mu_{2}\left(\alpha_{2}+\mu_{2}-\mu_{3}\right)^{2}}}{2 \sqrt{\mu_{2}} \sqrt{\mu_{3}}}$

- $\lambda_{6}=-\frac{\left(\alpha_{2}+\mu_{2}+\mu_{3}\right)}{2}+\frac{\sqrt{4 \beta_{2} \beta_{3} k_{2} k_{3}+\mu_{3} \mu_{2}\left(\alpha_{2}+\mu_{2}-\mu_{3}\right)^{2}}}{2 \sqrt{\mu_{2}} \sqrt{\mu_{3}}}$ 
- $\lambda_{7}=-\mu_{1}$

Observe que los valores propios $\lambda_{1}, \lambda_{2}, \lambda_{3}, \lambda_{4}, \lambda_{5} \mathrm{y} \lambda_{7}$ tienen parte real negativa y que el único valor propio que puede ser tanto positivo, negativo o cero es $\lambda_{6}$; entonces ¿para qué condición sobre los parámetros, el punto de equilibrio es estable o inestable? Observe que $\lambda_{6}<0$ implica que

$$
-\frac{\left(\alpha_{2}+\mu_{2}+\mu_{3}\right)}{2}+\frac{\sqrt{4 \beta_{2} \beta_{3} k_{2} k_{3}+\mu_{3} \mu_{2}\left(\alpha_{2}+\mu_{2}-\mu_{3}\right)^{2}}}{2 \sqrt{\mu_{2}} \sqrt{\mu_{3}}}<0,
$$

obteniendo la siguiente condición

$$
\frac{\beta_{2} \beta_{3} k_{2} k_{3}}{\mu_{2} \mu_{3}^{2}\left(\mu_{2}+\alpha_{2}\right)}<1
$$

Entonces, si el valor propio $\lambda_{6}$ cumple la condición (3), el equilibrio libre de infección es asintóticamente estable; mientras que si la condición (3) no se cumple, el punto de equilibrio libre de infección es inestable. En el caso del equilibrio endémico, no se pudo hacer un análisis de estabilidad de manera algebraica, sin embargo, en [4] se puede ver el análisis de estabilidad para dicho punto de equilibrio, hecho numéricemente.

Para continuar con el análisis del sistema, en la siguiente sección se hace el cálculo y el análisis de $R_{0}$.

\section{Cálculo y análisis de $R_{0}$ para el estudio de la Rickettsiosis}

En esta sección, se hará el clculo y el análisis del número reproductivo básico $\left(R_{0}\right)$, buscando, mediante dicho anlisis, estrategias que permitan reducir el valor de dicho parámetro. Finalmente haremos simulaciones del sistema (1) para los casos cuando $R_{0}<1$ y $R_{0}>1$.

El número reproductivo básico, $R_{0}$, es un parámetro umbral que en epidemiología es de suma importancia, ya que te permite tener información para determinar si se dará o no la propagación de una enfermedad. $R_{0}$ se define como el número de infecciones secundarias que produce un individuo infeccioso durante su periodo de infecciosidad, al ser introducido en una población totalmente susceptible. Este parámetro permite tener una idea de qué tan efectiva es una enfermedad al momento de propagarse. Es decir, es una medida promedio de cuántos individuos susceptibles a la enfermedad pueden ser contagiados por un individuo infeccioso. De aquí que $R_{0}$ es un parámetro umbral, en el sentido que si $R_{0}<1$ la enfermedad tiente a erradicarse con el tiempo, ya que un individuo infeccioso no alcanza a infectar a ningún individuo susceptible; por lo contrario, si $R_{0}>1$ la enfermedad se propagará, ya que cada individuo infeccioso puede infectar a más de un individuo susceptible. Así, entre mayor sea el valor de $R_{0}$, más rápido se propagará la enfermedad. Para el cálculo de este parámetro véase $[4,5]$.

Conociendo la expresión de $R_{0}$, la cual está en términos de los parámetros del sistema, se tiene la posibilidad de analizar qué parámetros se pueden manipular para reducir lo más posible su valor, es decir, se buscan medidas de control que permitan disminuir el valor de $R_{0}$ y así controlar de la mejor manera posible la propagación de la enfermedad en cuestin.

Así, haciendo los cálculos pertinentes, se puede probar que el número reproductivo básico para el sistema (1) está dado por: 


$$
R_{0}=\sqrt{\frac{\beta_{2} \beta_{3} k_{2} k_{3}}{\mu_{2}\left(\alpha_{2}+\mu_{2}\right) \mu_{3}^{2}}} .
$$

Podemos observar, a partir de la expresión de $R_{0}$, que la dinámica de propagación de la enfermedad está a cargo sólo de los perros y las garrapatas, ya que en la expresión de $R_{0}$ no participan los parámetros involucrados en la población de humanos. Esto corrobora que el humano es un hospedero accidental de la rickettsia.

$\mathrm{Al}$ analizar el modelo, podemos darnos cuenta que la dinámica de propagación de la enfermedad se da a través del ciclo de infección entre los perros y las garrapatas. Este hecho se ve reflejado en la expresión de $R_{0}$, es decir, reescribiendo la expresión de $R_{0}$ se puede distinguir la aportación de cada población a la propagación de la enfermedad.

De esta manera, podemos reescribir a $R_{0}$ de la siguiente forma: $R_{0}=\sqrt{\frac{\beta_{2} k_{2}}{\mu_{2}\left(\alpha_{2}+\mu_{2}\right)} \cdot \frac{\beta_{3} k_{3}}{\mu_{3}^{2}}}$, donde $\frac{\beta_{2} k_{2}}{\mu_{2}\left(\alpha_{2}+\mu_{2}\right)}$ es el número de garrapatas infectadas que produce un perro infectado, durante su período de infecciosidad y $\frac{\beta_{3} k_{3}}{\mu_{3}^{2}}$ es el número de perros infectados que produce una garrapata infectada durante su período de infecciosidad.

Ya que hemos obtenido a $R_{0}$, lo que buscaremos es reducir el valor del mismo. Esto se hace con el fin de implementar medidas de control para que la enfermedad no se propague dentro de la población. En nuestro caso, propondremos medidas de control para reducir la propagación de la rickettsia rickettsii.

Observemos que para reducir el valor de $R_{0}$, lo podemos conseguir de dos maneras: reducir el valor de $\beta_{2} \beta_{3} k_{2} k_{3}$, o bien, aumentando el valor de $\mu_{2}\left(\alpha_{2}+\mu_{2}\right) \mu_{3}^{2}$.

Entonces para reducir el valor de $\beta_{2} \beta_{3} k_{2} k_{3}$, lo conseguimos reduciendo el valor de los parámetros $\beta_{2}, \beta_{3}, k_{2}$ ó $k_{3}$. Para ello, biológicamente implica disminuir la tasa de infección de las garrapatas infectadas a los perros susceptibles, la tasa de infección de los perros infectados a las garrapatas susceptibles y las tasas de reclutamiento de perros y garrapatas, respectivamente.

Analizando la viabilidad de reducir $k_{2}$ y $k_{3}$ tenemos que, para el parámetro $k_{2}$ es complicado reducir su valor, ya que en el modelo de la rickettsiosis solo hemos considerado a los nacimientos de los perros domésticos y no a los que se están produciendo en las calles. Una de las medidas para reducir los nacimientos de los perros es esterilizarlos, pero, por la misma razón de que hay muchos en las calles, esto no resultaría muy útil y no muchas de las personas están dispuestas a esterilizar a sus perros.

Por otro lado, el parámetro $k_{3}$ es casi imposible de disminuir. Por una parte, podemos disminuir la población de garrapatas que están afectando a los perros domésticos pero en los perros de la calle no, ya que ellos no se les proporciona una higiene adecuada y, por lo tanto, tienden a estar más en contacto con las garrapatas y de esta manera se pueden alimentar hasta llegar a reproducirse depositando una cantidad muy grande de huevos, que para la vista humana no son fáciles de detectar. Otro factor que influye es que no estamos pensado en las garrapatas que también están en la tierra, pastizales, entre otros lugares, y por lo tanto no podemos detectar a las crías pequeñas, tales como larvas y ninfas, que también pueden transmitir la enfermedad, e incluso a los huevos que cada garrapata hembra puede depositar en el medio ambiente.

Para disminuir los parámetros $\beta_{2}$ y $\beta_{3}$, notemos primero que el parámetro $\beta_{3}$ podemos disminuirlo dando baños con jabones antigarrapatas para que éstas mueran al momento de picar a 
algún perro o dejen de alimentarse del mismo, reduciendo la población de garrapatas que están y no están infectadas, ya que su única forma de infectarse de la rickettsia rickettsii es cuando se alimentan de los perros infectados.

Para disminuir el parámetro $\beta_{2}$, podemos vacunar a los perros susceptibles con el fin de que adquieran inmunidad a la rickettsia rickettsii y que la enfermedad no los afecte al momento de que una garrapata pueda estar alimentándose de él. En el caso de que los perros ya estén infectados, se debe empezar el tratamiento para que el perro pueda curarse y además adquiera inmunidad a la rickettsia.

Por otro lado, para aumentar el valor de $\mu_{2}\left(\alpha_{2}+\mu_{2}\right) \mu_{3}^{2}$, lo conseguimos matemáticamente aumentando los valores de $\mu_{2}, \alpha_{2}$ ó $\mu_{3}$, pero biológicamente, aumentar el parámetro $\mu_{2}$ implica aumentar la rapidez con la que mueren los perros, lo cual queremos evitar, ya que implicaría sacrificar de alguna manera a los perros.

Aumentar el valor del parámetro $\mu_{3}$ implica, al igual que en el caso de los perros, provocar que el periodo de vida de las garrapatas sean menores, lo cual resulta muy difícil; esto porque no es sencillo detectar a las garrapatas que no están alimentándose en el perro y para aquellas garrapatas que están en el medio ambiente se podrían utilizar pesticidas para hacer que las garrapatas del medio ambiente mueran, pero esto será muy difcil, ya que no hay una zona bien definida donde estén las garrapatas. Por lo que no podremos aumentar los parámetros $\mu_{2} \mathrm{y} \mu_{3}$.

El parámetro $\alpha_{2}$ representa la tasa de muerte por la enfermedad para los perros. Aumentar este parmetro significaría que debemos infectar a los perros para que murieran por la enfermedad, lo cual no queremos que pase, por lo que, biológicamente no podemos aumentar los parámetros $\mu_{2}$, $\alpha_{2}$ ni $\mu_{3}$.

Después de este análisis de los parámetros, podemos observar que una forma biológicamente aceptable para reducir el valor de $R_{0}$ consiste en disminuir las tasas de infección representadas por $\beta_{2}$ y $\beta_{3}$.

Así, una medida de prevención para que los perros que no están infectados no se infecten o que las infecciones sean las mínimas posibles, es vacunarlos contra la rickettsia rickettsii. Esto provocará en aquellos perros que no estén infectados una inmunidad temporal o total hacia la enfermedad y, cuando lo hagamos en los perros que están vacunados, estos tenderán disminuir, ya que con la vacuna van a poder recuperarse y, después de que se hayan recuperado, van a adquirir una inmunidad temporal o total contra la enfermedad.

Otra medida para poder cesar la enfermedad es, para aquellos perros que estén infectados, tratarlos con el medicamento debido para que los síntomas y la enfermedad vayan disminuyendo en los perros infectados y, de esta manera, la población de perros que están infectados disminuya, haciendo que la propagación de la enfermedad disminuya. También, mientras el tratamiento dura y después que haya concluido y los perros se hayan curado de la enfermedad, para que estos no sean atacados por las garrapatas de nuevo, hay que darles un tratamiento continuo anti garrapatas para que los perros estén más protegidos y así la enfermedad tampoco se propague.

Con el tratamiento dado a los perros infectados y las vacunas a los que están y no están infectados, la población de garrapatas infectadas debe disminuir con el paso del tiempo, con lo cual se disminuirían las infecciones de las garrapatas provocando que la propagación de la enfermedad vaya disminuyendo. 


\subsection{Simulación del modelo para la Rickettsia}

En esta sección, ilustraremos con aproximaciones de las soluciones del sistema (1) el comportamiento de las poblaciones que participan en dicho modelo cuando están en presencia de la enfermedad. Se presentarán simulaciones para valores de los parámetros que generen $R_{0}<1$ y también $R_{0}>1$.

En la figura 3 podemos observar el comportamiento de las poblaciones de humanos y perros para $R_{0}=0.517$.

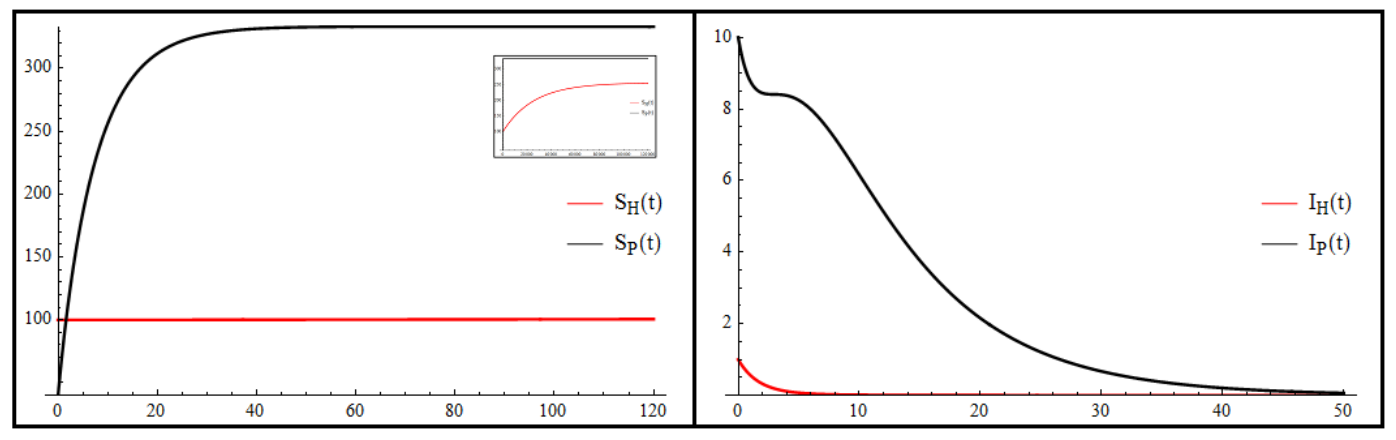

Figura 3: Comportamiento de las poblaciones de humanos y perros para $R_{0}=0.517$.

En esta gráfica podemos observar que el comportamiento, tanto en humanos como en los perros, es el esperado para los valores de los parámetros usados. Ambas clases de susceptibles tienden a su punto de equilibrio; en particular observe que la población de perros susceptibles tiende a $\frac{k_{2}}{\mu_{2}}$ con el paso del tiempo, lo cual representa la población máxima de perros. En el caso de la población de humanos susceptibles, observe que prácticamente no cambia la dinámica durante un periodo muy grande de tiempo, sin embargo, para valores de $t$ muy grandes, la población de humanos susceptibles alcanzan el punto de equilibrio. Este comportamiento en la población de humanos susceptibles, confirma que la población de humanos no interviene mucho en la dinámica de propagación de la enfermedad. En la gráfica también las poblaciones de infectados tienden rápidamente a cero.

En la figura 4 se presentan las simulaciones del comportamiento de la población de las garrapatas para $R_{0}=0.517$.

Para el caso de las garrapatas, podemos observar que con el paso del tiempo la población de garrapatas susceptibles crece y tiende a $\frac{k_{3}}{\mu_{3}} \mathrm{y}$, en el caso de las garrapatas infectadas, se observa que la población tiende a cero. 


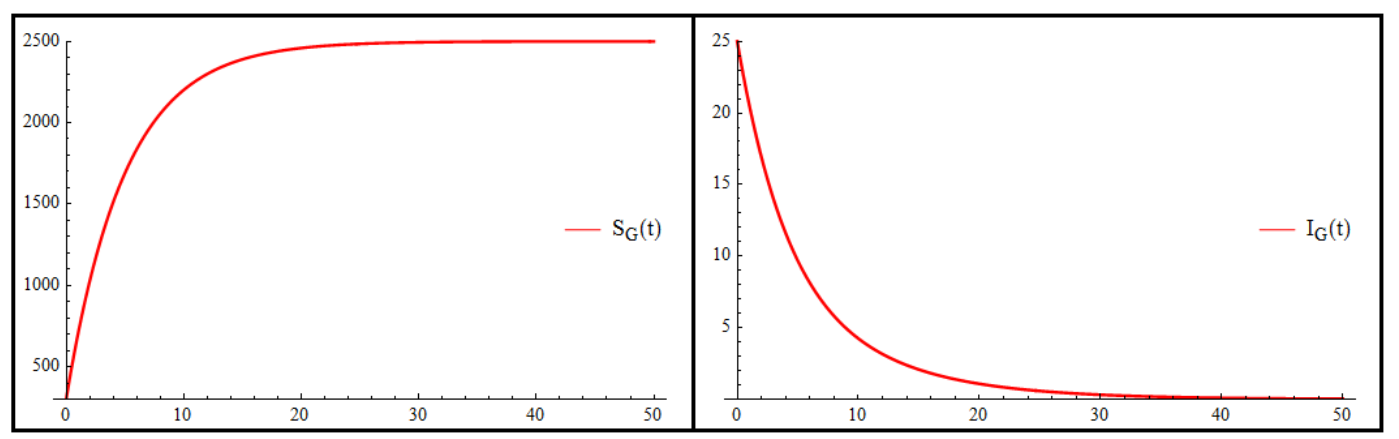

Figura 4: Comportamiento de la población de garrapatas para $R_{0}=0.517$.

Como podemos observar, cuando tenemos un $R_{0}=0.517$, esperábamos que las poblaciones infectadas decayeran y así sucedió. Esto nos indica que cuando tenemos un $R_{0}<1$ la enfermedad, con el paso del tiempo, desaparecerá.

Ahora observemos la simulación cuando tenemos que $R_{0}>1$. Para ello, consideramos un $R_{0}=1.89$, el cual lo hemos obtenido utilizando un conjunto de parámetros para los cuales el equilibrio libre de infección es inestable y el equilibrio endémico estable. Este conjunto de parámetro genera un cambio de estabilidad en el punto de equilibrio libre de infección.

En la figura 5, se presenta el comportamiento de la poblaciones de humanos y perros para $R_{0}>1$.

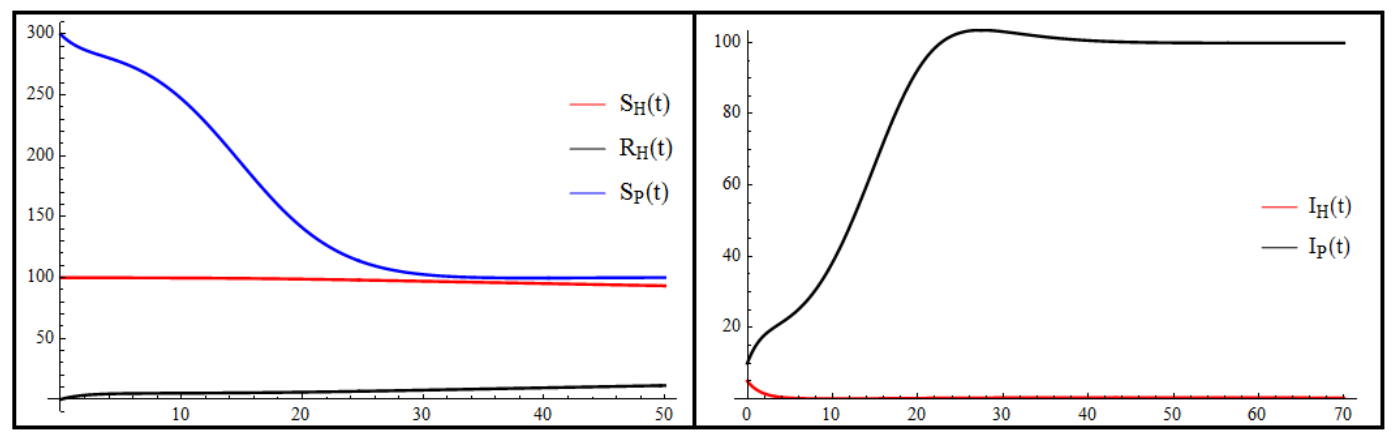

Figura 5: Comportamiento de las poblaciones de humanos y perros para $R_{0}=1.89$.

Para $R_{0}=1.89$, la población de perros susceptible también tiende a decrecer y se estabiliza en un determinado valor menor a la población inicial. En el caso de las poblaciones de humanos susceptibles y recuperados, se observa de nuevo que se requiere de mucho tiempo para que alcancen sus equilibrios. Respecto a los perros infectados, podemos observar que su número va creciendo mediante un comportamiento análogo al logístico, hasta alcanzar la estabilidad; mientras que la población de humanos infectados, aunque $R_{0}>1$, se observa que decrece ya que el equilibrio para dicha variable está muy cerca de cero, un indicio más de que es un hospedero accidental.

Finalmente, en la figura 6, presentamos el comportamiento de la población de las garrapatas para $R_{0}>1$. 


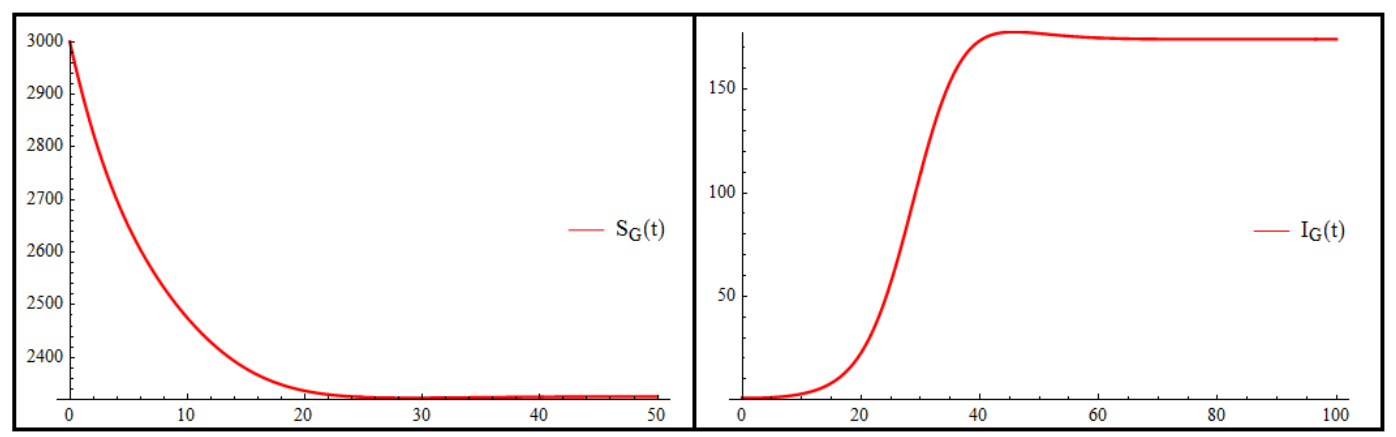

Figura 6: Comportamiento de la población de garrapatas para $R_{0}=1.89$.

Podemos observar que las población de garrapatas susceptibles decrece de manera exponencial, mientras que la población de garrapatas infectadas tiene un crecimiento de tipo logístico, alcanzando su estabilidad en un tiempo relativamente corto.

\section{Conclusión}

Se propuso y se analizó un modelo SIR para el estudio de la zoonosis Rickettsiosis. Se hizo un estudio de estabilidad del punto de equilibrio libre de infección, se calculó su número reproductivo básico, en el cual se pudo observar que los humanos no intervienen en la dinámica de transmisión de la enfermedad. En base al análisis del número reproductivo básico se propusieron medidas de control que permiten la reducción de $R_{0}$, de todas ellas, la más viable, tanto biológica como económicamente es la de reducir $\beta_{2}$, lo que implica tratar a la población de perros, ya sea a través de vacunas, baños garrapaticidas o algún otro tratamiento, para que los perros adquieran inmunidad a la rickettsia y que no puedan propagarla a las garrapatas y así disminuir la incidencia de la enfermedad. Se hizo un estudio numérico del modelo, el cual permite corroborar la estabilidad de los puntos de equilibrio, pero ahora en términos de $R_{0}$ y se presentan simulaciones sobre el comportamiento de las distintas poblaciones que participan en el modelo para valores de $R_{0}$ mayor y menor que uno. 


\section{Referencias}

[1] Barba E., José R.,Fiebre manchada de las Montañas Rocosas, Unidad Médica de Alta Especialidad: Lic. Ignacio García Téllez, Instituto Mexicano del Seguro Social. Mérida, Yucatán., www.medigraphic.org.mx, Recibido: 09/07/2009, Aceptado:13/07/2009.

[2] Quintero V., Juan C., Hidalgo, Marylin, Rodas G. Juan D., Rickettsiosis, una enfermedad letal emergente y re-emergente en Colombia, Universitas.Scientiarum, 2012, Vol. 17 N. 1: 82-99.

[3] Mercado U., Mónica C, Rickettsiosis. Historia y actualidades, Enf Inf Microbiol 201030 (1): 25-31, fecha de aceptación: agosto 2009.

[4] Gabriel Adrián Salcedo Varela, Número Reproductivo Básico Para Sistemas Epidemiológicos: Una Aplicación al Estudio de la Rickettsiosis, Hermosillo, Sonora (2015), editorial Universidad de Sonora. Unidad Regional Centro. División de Ciencias Exactas y Naturales, pp. 1-103, tesis de licenciatura en matemáticas.

[5] P. van den Driessche, James Watmough, Reproduction numbers and sub-threshold endemic equilibria for compartmental models of disease transmission, Math. Biosci., 180 (2002). 